\title{
A HYBRID APPROACH FOR POLARITY SHIFT DETECTION
}

\author{
Michele Mistry ${ }^{1}$ and Prem Balani ${ }^{2}$ \\ Department of Information Technology, G.H. Patel College of Engineering and Technology, India
}

\begin{abstract}
Now-a-days sentiment analysis has become a hot research area. With the increasing use of internet, people express their views by using social media, blogs, etc. So there is a dire need to analyze people's opinions. Sentiment classification is the main task of sentiment analysis. But while classifying sentiments, the problem of polarity shift occurs. Polarity shift is considered as a very crucial problem. Polarity shift changes a text from positive to negative and vice versa. In this paper, a hybrid approach is proposed for polarity shift detection of negation (explicit and implicit) and contrast. The hybrid approach consists of a rule-based approach for detecting explicit negation and contrast and a lexicon called SentiWordNet for detecting implicit negation. The proposed approach outperforms its baselines.
\end{abstract}

\section{Keywords \\ Sentiment Analysis, Sentiment Classification, Polarity Shift, Natural Language Processing, Lexicon}

\section{INTRODUCTION}

In recent years the use of internet and e-commerce has increased. More and more products are sold on the Web, and the people buying the products write reviews on it. The data that Web contains is in the form of product reviews, news, blogs, internet forums etc. The volume of online reviews available on the Internet is growing day by day. As a result of this growth, sentiment analysis has become a hot field in the area of natural language processing. Natural language processing (NLP) is actually a theory-motivated range of computational techniques that are used for the automatic analysis and representation of human language [34].The term sentiment analysis first appeared in the work of Nasukawa and Yi [30]. The term opinion mining first appeared in the work of Dave, Lawrence and Pennock [29]. But the research on sentiments and opinions appeared much earlier [2], [8], [9], [31], [32], [33]. In literature, subjectivity and emotion are closely related to sentiment and opinion. An objective sentence is the sentence that represents some factual information about the world. Whereas, a subjective sentence expresses some feelings, views or beliefs [1].

Objective Sentence: "iPhone is an Apple product."

Subjective Sentence: "I like iPhone."

Sentiments consist of feelings, thoughts and emotions of an individual for a particular event or topic [11]. According to Bing Liu [1], "Sentiment analysis, also called opinion mining, is the field of study that analyzes people's opinions, sentiments, evaluations, appraisals, attitudes, and emotions towards entities such as products, services, organizations, individuals, issues, events, topics, and their attributes. And the most important indicators of sentiments are sentiment words or opinion words."

The basic task in sentiment analysis is sentiment classification. Sentiment classification is considered to be a topic that is studied very extensively in recent times [7]. Sentiment classification is used to classify the text as positive, negative or neutral [1]. While doing sentiment classification, many times there occurs a problem called the polarity shift problem. Detecting the polarity shifts is a very important task for sentiment classification. Polarity shift is considered to be a linguistic phenomenon. In polarity shift, the polarity of a particular review changes from positive to negative or vice versa [3]. While doing sentiment classification, if the Bag-of-words (BOW) model is used then it will not regard any grammar, as a result of that the syntactic structure of the sentence may be disturbed and that'll cause problems like polarity shift detection, anaphora resolution, etc. [6]. The words that change the polarity of the text are called polarity shifters. They are also called "valence shifter" [5] and "Sentiment shifter" [1]. There are many kinds of polarity shifters like explicit and implicit negation, contrast, likelihood, counter factual, etc.

By detecting the polarity shifters, one can know the reason that has changed the polarity of the text. For e.g. "I am not satisfied with the working of this juicer." In the above review, "not" is the polarity shifter that is changing the polarity of the review to negative.

The linguistic phenomenon in which the sentimental orientation of the whole text is changed from negative to positive or vice versa; is called polarity shifting [3], [4]. Polarity shift is caused by polarity shifters, e.g., contrast, negation, likelihood, etc. [6]. Negation is considered as the most common type of polarity shift. Negation is of two types: explicit negation and implicit negation. Explicit negators are words like "no", "not" etc. Implicit negators are words like "avoid", "deny" etc. The various approaches that are used for polarity shift detection are: machine learning approaches [2], [8], lexicon-based approaches [11], [13], [17] and hybrid approaches [18]. These are widely used along with some rule based approaches and statistical approaches.

This paper presents a hybrid approach for polarity shift detection. A rule-based method for detecting the explicit negators and contrasts is used. An updated negation list using SentiStrength lexicon has been used. SentiWordNet has been used to detect implicit negators and compare the obtained results with baseline approaches.

The rest of the paper is organized as follows: Section 2 contains the related work. Section 3 contains the proposed work, section 4 contains the experimental results and Section 5 contains the conclusion.

\section{RELATED WORK}

Sentiment analysis is a growing research field [3]. According to Bing Liu [1], "Sentiment analysis is considered as a highly restricted NLP problem. It is so because there is no need for the system to fully understand the semantics of each sentence or document but it only needs to understand some aspects of it, i.e., positive or negative sentiments and their target entities or topics." 
Sentiment classification is considered as the basic task of sentiment analysis [4]. The goal of sentiment classification is to classify the sentiments. E.g. positive, negative and neutral. The methods used for sentiment classification are: machine learning approaches [2],[8], lexicon-based approaches[11],[13],[17] and hybrid approaches[18].

According to Yi, Jeonghee, et al. [35], the two challenging aspects of sentiment analysis are: "Even though the overall opinion about a topic is useful; only a part of information is of interest. The document level sentiment classification fails to detect sentiment about individual aspects of the topic. And associating the extracted sentiment to a specific topic is difficult." But in [29], according to Dave, Kushal, Steve Lawrence, and David M. Pennock; most of the statistical algorithm perform poorly in this respect.

In the machine learning approach, classifiers are built from labeled instances of texts or sentences, which is the supervised classification task [2]. Machine learning approaches need trained data and they are domain specific. Lexicon based methods make use of pre-built lexicons of words weighted with their sentiment orientations to determine the overall sentiment of a given text [15]. They don't need training data. And can be used for a wide range of domains [15]. Sentiment classification can be performed at following levels:

\section{- Document Level}

- Sentence Level

- Phrase Level

\section{- Aspect Level}

The task at document level is to classify whether a whole opinion document expresses a positive or negative sentiment [2]. The task at sentence level is to determine whether each sentence expresses a positive, negative or neutral opinion. According to Bing Liu [1], "There is no fundamental difference between document and sentence level classifications because sentences are just short documents. But some researches often make assumptions about sentence-level analysis. One assumption is that a sentence usually contains a single opinion (although not true in many cases)." A document contains multiple opinions. The task at phrase level is to determine the opinion that is being expressed by a phrase [28]. Aspect level performs fine-grained analysis. Aspect level directly looks at the opinion itself. It is based on the idea that an opinion consists of a sentiment (positive or negative) and a target (of opinion) [1].

Document level and sentence level sentiment classification is considered very challenging. The aspect level is considered as even more difficult than these two [1]. The aspect level classification is fine-grained.

In literature, many attempts have been made to solve the sentiment classification problem. Polarity shifting is a challenging problem in sentiment classification. It plays a crucial role in sentiment classification. Its focus is on subjectivity analysis with changing polarities, intensities and ranking [37]. In polarity shifting the sentiment orientation of the whole text is different from its containing words [10]. In literature, some researchers have focused on polarity analysis where an opinion is regarded as highly positive, positive, negative or highly negative [38]. Whereas some others have worked on human emotions e.g. happy or sad to take appropriate actions from the analyzed content [39].
Usually it is difficult to capture the sentiment reversion caused by polarity shifters in the BOW model, as two sentiment-opposite texts (e.g., "I am happy with this phone" and "I am not happy with this phone") are regarded to be very similar in the BOW representation [3]. The two main steps for considering polarity shift are: 1) Detecting polarity shifted words or sentences. 2) Designing specific classification algorithms. The different kinds of polarity shifters should be handled in different ways. According to [3], "Negation is able to shift the sentiment polarity within the phrase." The early work on polarity shifting focuses on negation [2], [11], [26], [21]

According to Li et al. [6], the polarity shifters are distributed as shown in the Table.1. The graphical depiction Table.1 is also shown. The Fig.1 shows the distribution of the polarity shifters according to the statistics conducted by Li et al. in [6].

In literature, there are two main types of methods for document and sentence level: term counting methods and machine learning methods [4]. In term counting methods the scores of the content words or phrases are summed up and the overall sentiment is obtained, according to the manually-collected or external lexical dictionaries [8], [27]. Whereas in machine learning methods, a piece of text is represented by a bag-of-words (BOW) model and then machine learning algorithms are used for classification [2]. Generally the machine learning methods are considered to be more effective for sentiment classification [36]. But, it is not easy to handle polarity shift based on the BOW model [36]. The way to handle polarity shift is different for both the methods. In [11], [14], [23], [24]; for aspect level sentiment analysis, polarity shift problem was considered by both corpus and lexicon based method.

The term-counting methods are relatively easy to modify to include polarity shift. This is done by directly reversing the sentiment of polarity-shifted words and then summing up the sentiment score word by word [5], [20], [25], [26]. In literature, machine learning methods are more widely used compared to term counting methods for sentiment classification. But integrating the information about polarity shift into BOW model in such methods is difficult. And they achieve much better performance compared to term counting approaches [2], [26]. They have also been adopted for complicated scenarios like domain adaption [19], multi-domain learning [16] and semi-supervised learning [12], [22] for sentiment classification.

Table.1. Statistics on various polarity shifting structures

\begin{tabular}{|c|c|c|}
\hline $\begin{array}{c}\text { Polarity Shifting } \\
\text { Structures }\end{array}$ & $\begin{array}{c}\text { Trigger } \\
\text { Words/Phrases }\end{array}$ & $\begin{array}{c}\text { Distribution } \\
\text { (\%) }\end{array}$ \\
\hline Explicit Negation & not, no, without & 37 \\
\hline Contrast Transition & $\begin{array}{c}\text { but, however, } \\
\text { unfortunately }\end{array}$ & 20 \\
\hline Implicit Negation & avoid, hardly & 7 \\
\hline False Impression & look, seem & 6 \\
\hline Likelihood & probably, perhaps & 5 \\
\hline Counter-factual & should, would & 5 \\
\hline Exception & the only & 5 \\
\hline Until & until & 3 \\
\hline
\end{tabular}

Many approaches have been proposed for polarity shift detection. They are: Lexicon-based and machine learning 
approaches. Some researchers have proposed rule based and statistical based approaches, whereas some are using dual training algorithm, term counting algorithm with polarity shifting and sentence polarity shift algorithm. But according to [3], [7] the improvement is less, as the different kinds of polarity shifters have to be considered.

\section{PROPOSED WORK}

The proposed hybrid approach uses two techniques; a lexicon called SentiWordNet and a Rule-based approach. As hybrid approaches are combination of approaches; they have more advantage. The polarity shifters are considered with negators (explicit and implicit) and contrast. Because negation and contrast are the polarity shifters that are mostly found in a review text [2][5].

Explicit negators are negators that can be easily detected. Explicit negators have explicit hint. E.g. "I am not satisfied with this product." Here the word "not" is the explicit negator. Implicit negators don't have any explicit hint. The context behind the word should be known while detecting implicit negators. e.g.

1. "Buying this product is waste of money."

2. "Throw the waste in the dustbin."

In example 1, "waste" is an implicit negator as it does not give an explicit hint. But in example 2, the word "waste" has been used in context with the word "rubbish".

The examples for contrast are words like "but", "however", "yet", "unfortunately", "though", "although" and "nevertheless". E.g. I like the phone but I hate it's color". In this example, "but" indicates contrast.

The Fig. 1 shows the proposed approach First the datasets have been preprocessed. The various parts of speech have been detected and some stops words have been eliminated. Stop words are those words that convey no meaning on some text. E.g. "The who". Here there is no need of "the" before "who". So "the" has to be eliminated. The hybrid approach consists of a rule-based approach and a lexicon called SentiWordNet. Normally SentiWordNet uses unigram features but in this approach SentiWordNet has been incorporated with bigram features. E.g. "I am not unhappy". In this example, if unigram features are considered then just a single word will be considered like $\{\mathrm{I}\},\{\mathrm{am}\},\{$ not $\},\{$ unhappy $\}$. But if bigram features are considered then the review can be divided as $\{\mathrm{I}, \mathrm{am}\}$, \{am, not\}, \{not, unhappy\}. As it is seen, "not unhappy" means "happy" but if unigram features are considered the words "not" and "unhappy" will be considered separately and the review will be called negative but actually it is positive. Because of this reason we've used bigram features. As bigram features will detect not, unhappy\} together and thereby the review will be correctly classified as a positive review. This is helpful for improving the accuracy. Furthermore, a Rule-based approach has been used for identifying the explicit and implicit negators. The list of explicit negators given in [3] has been updated by using SentiStrength [42] lexicon. The list contains negators like \{"no", "not", "n't", "none", "nobody", "nothing", "never", "hardly", "seldom" and "without", "aren't", "cannot", "cant", "couldnt", "dont", “isnt", “wont", "wouldnt" $\}$. SentiStrength has been used as it is very useful to detect polarity shifters from twitter data. Finally all the polarity shifters (negation and contrast) have been extracted from the reviews.

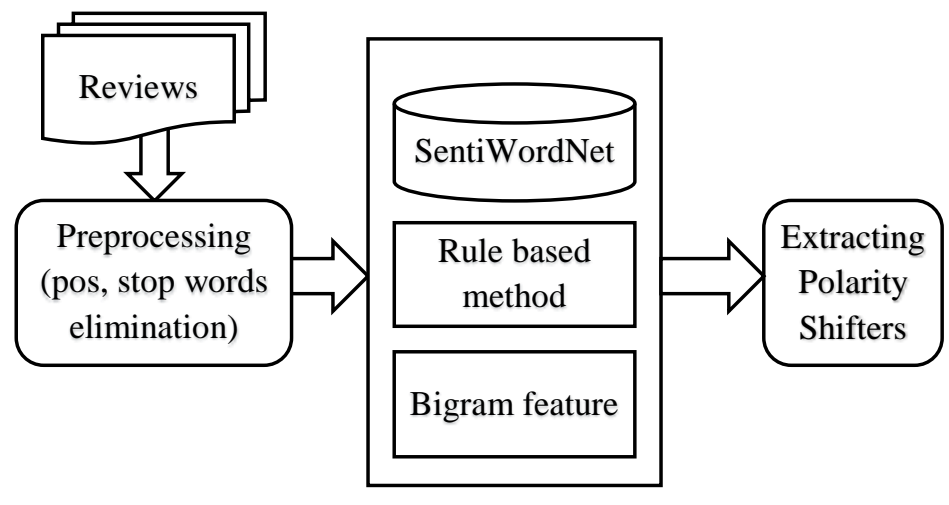

Fig.1. Proposed Approach

\section{EXPERIMENTAL STUDIES}

\subsection{EXPERIMENTAL SETUP}

Both the baselines and the proposed approach using java technology is evaluated on two different datasets. The first one is Cornell movie reviews [40]. It contains total 10,662 reviews, out of which 5331 positive and 5331 negative snippets/sentences. The second dataset is the Healthcare Reform (hcr) dataset [41]. It contains tweets with \#hcr hashtag on twitter. It is a type of twitter dataset. It has 839 annotated reviews out of which 172 are positive, 464 are negative and 204 are neutral.

\subsection{EXPERIMENTAL RESULTS}

Both baselines and approach is evaluated using SentiWordNet with bigram features and rule-based approach with updated list of negators using SentiStrength. The results obtained from evaluating baselines and the proposed approach have been compared with each other. For baselines, SentiWordNet lexicon, Rule-based approach for detecting just negators and rule-based polarity shift approach were considered. The results are shown in the Table.2.

Table.2. Performances of baselines and proposed approach

\begin{tabular}{|l|c|c|}
\hline Methods & $\begin{array}{c}\text { Accuracy for Cornell } \\
\text { movie reviews }\end{array}$ & $\begin{array}{c}\text { Accuracy for } \\
\text { her }\end{array}$ \\
\hline SentiWordNet & $58.92 \%$ & $51.2 \%$ \\
\hline Rule-based & $53.15 \%$ & $34 \%$ \\
\hline $\begin{array}{l}\text { Rule-based polarity } \\
\text { shift }\end{array}$ & $67.3 \%$ & $53.9 \%$ \\
\hline HAPSD & $71.81 \%$ & $61.27 \%$ \\
\hline
\end{tabular}

The proposed approach outperforms the baseline approaches (SentiWordNet, Rule-based approach and Rule-based approach for polarity shift detection of negation and contrast). The performance is calculated using the following formula:

$$
\text { Accuracy }=(t p+t n) /(t p+t n+f p+f n)
$$

where, $t p$ - true positive, $t n$ - true negative, $f p$ - false positive, $f n$ false negative.

The comparative analysis of the proposed approach with the baselines is shown in Fig.2. It can be seen that the proposed hybrid approach outperforms the baseline approaches. 


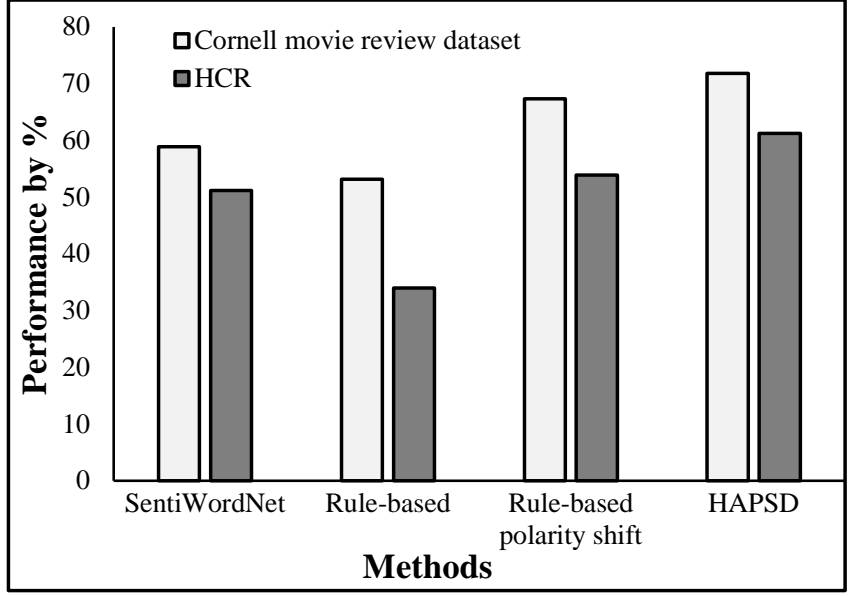

Fig.2. Comparative Results of HAPSD with Baselines by Bar Graph

\section{CONCLUSION}

This paper proposes a hybrid approach for detecting polarity shifters. The field that analyzes people's opinions and feelings, is called sentiment analysis (opinion mining). Sentiment classification is the main task in sentiment analysis and while classifying using BOW model, the problem of polarity shift occurs. Polarity shift is considered to be a very challenging problem for sentiment classification. It is a type of linguistic phenomenon that changes the text from positive to negative and vice versa. Till now, many approaches like machine learning, lexicon-based and hybrid; have been proposed for sentiment classification and polarity shifting. But researchers say that despite of so many approaches for polarity shift detection, the improvement is very less.

As a result, there is need to develop more approaches. Because there are different types of polarity shifters and each shifter has to be handled in its own way. The proposed approach is a hybrid approach for polarity shift detection. A lexicon called SentiWordNet and a rule-based method have been used. SentiWordNet has been used for detecting implicit negators and rule-based approach for detecting explicit negators and contrast. Bigram features have been incorporated in SentiWordNet. The negation list of the rule-based approach has been updated using a lexicon called SentiStrength. Because of this, polarity shifters in twitter-like data that lack proper grammar can be detected. The proposed approach has been compared with baselines (SentiWordNet, rule-based approach and rule-based approach for polarity shift detection of negators and contrast). It gives higher accuracy as compared to the accuracy of the baselines. The accuracy for Cornell movie review dataset is $71.81 \%$ and for hor dataset, it is $61.27 \%$.

\section{REFERENCES}

[1] Bing Liu, "Sentiment Analysis and Opinion Mining Synthesis Lectures on Human Language Technologies", Morgan and Claypool Publishers, 2012.

[2] Bo Pang, Lillian Lee and Shivakumar Vaithyanathan. "Thumbs up? : Sentiment Classification using Machine Learning Techniques", Proceedings of ACL Conference on
Empirical Methods in Natural Language Processing, Vol. 10, pp. 79-86, 2002.

[3] Rui Xia et al., "Polarity Shift Detection, Elimination and Ensemble: A Three-Stage Model for Document-Level Sentiment Analysis", Information Processing and Management, Vol. 52, No. 1, pp. 36-45, 2016.

[4] Rui Xia et al., "Dual Sentiment Analysis: Considering Two Sides of One Review", IEEE Transactions on Knowledge and Data Engineering, Vol. 27, No. 8, pp. 2120-2133, 2015.

[5] Livia Polanyi and Annie Zaenen, "Contextual Lexical Valence Shifters", Proceedings of the AAAI Spring Symposium on Exploring Attitude and Affect in Text, pp. 16, 2004.

[6] Shoushan Li et al., "Sentiment Classification and Polarity Shifting", Proceedings of the $23^{\text {rd }}$ International Conference on Computational Linguistics Association for Computational Linguistics, pp. 635-643, 2010.

[7] Shoushan Li, Zhongqing Wang, Sophia Yat Mei Lee and Chu-Ren Huang, "Sentiment Classification with Polarity Shifting Detection", Proceedings of IEEE International Conference on Asian Language Processing, pp. 129-132, 2013.

[8] Peter D. Turney, “Thumbs Up or Thumbs Down? Semantic Orientation Applied to Unsupervised Classification of Reviews", Proceedings of $40^{\text {th }}$ Annual Meeting on Association for Computational Linguistics Association for Computational Linguistics, pp. 417-424, 2002.

[9] Sanjiv Das and Mike Chen, "Yahoo! for Amazon: Extracting Market Sentiment from Stock Message Boards", Proceedings of the Asia Pacific Finance Association Annual Conference, Vol. 35, pp. 1-6, 2001.

[10] Xiaoqian Zhang, Shoushan Li, Guodong Zhou and Hongxia Zhao, "Polarity Shifting: Corpus Construction and Analysis", Proceedings of International Conference on Asian Language Processing, pp. 272-275, 2011.

[11] Xiaowen Ding, Bing Liu and Philip S. Yu, "A Holistic Lexicon-based Approach to Opinion Mining", Proceedings of ACM International Conference on Web Search and Data Mining, pp. 231-240, 2008.

[12] Xiaojun Wan, "Co-Training for Cross-Lingual Sentiment Classification", Proceedings of the Joint Conference $47^{\text {th }}$ Annual Meeting of the ACL and $4^{\text {th }}$ International Joint Conference on Natural Language Processing, Vol. 1, pp. 235-243, 2009.

[13] Maite Taboada et al., "Lexicon-based Methods for Sentiment Analysis", Computational Linguistics, Vol. 37, No. 2, pp. 267-307, 2011.

[14] Xiaowen Ding and Bing Liu, "The Utility of Linguistic Rules in Opinion Mining", Proceedings of $30^{\text {th }}$ Annual International ACM SIGIR Conference on Research and Development in Information Retrieval, pp. 811-812, 2007.

[15] Hassan Saif, Miriam Fernandez, Yulan He and Harith Alani, "Senticircles for Contextual and Conceptual Semantic Sentiment Analysis of Twitter", European Semantic Web Conference, pp. 83-98, 2014.

[16] Shoushan Li and Chengqing Zong, "Multi-Domain Sentiment Classification", Proceedings of $46^{\text {th }}$ Annual Meeting of the Association for Computational Linguistics on Human Language Technologies: Short Papers, pp. 257-260, 2008. 
[17] Andrea Esuli and Fabrizio Sebastiani, "Sentiwordnet: A Publicly Available Lexical Resource for Opinion Mining", Proceedings of $5^{\text {th }}$ Conference on Language Resources and Evaluation, pp. 417-422, 2006.

[18] Orestes Appel et al., "A Hybrid Approach to the Sentiment Analysis Problem at the Sentence Level", Knowledge-Based Systems, Vol. 108, pp. 110-124, 2016.

[19] John Blitzer, Mark Dredze and Fernando Pereira, "Biographies, Bollywood, Boom-Boxes and Blenders: Domain Adaptation for Sentiment Classification", Proceedings of $45^{\text {th }}$ Annual Meeting of the Association of Computational Linguistics, pp. 440-447, 2007.

[20] Minqing $\mathrm{Hu}$ and Bing Liu, "Mining and Summarizing Customer Reviews", Proceedings of $10^{\text {th }}$ ACM International Conference on Knowledge Discovery and Data Mining, pp. 168-177, 2004.

[21] Jin-Cheon Na et al., "Effectiveness of Simple Linguistic Processing in Automatic Sentiment Classification of Product Reviews", Proceedings of International Conference on International Society for Knowledge Organization, pp. 4954, 2004.

[22] Sajib Dasgupta and Vincent Ng, "Mine the Easy, Classify the Hard: A Semi-Supervised Approach to Automatic Sentiment Classification", Proceedings of Joint Conference on $47^{\text {th }}$ Annual Meeting of the ACL and the $4^{\text {th }}$ International Joint Conference on Natural Language Processing, pp. 701709, 2009.

[23] Minqing $\mathrm{Hu}$ and Bing Liu, "Mining Opinion Features in Customer Reviews", American Association for Artificial Intelligence, Vol. 4, No. 4, pp. 755-760, 2004.

[24] Xiaowen Ding, Bing Liu and Lei Zhang, "Entity Discovery and Assignment for Opinion Mining Applications", Proceedings of $15^{\text {th }}$ ACM International Conference on Knowledge Discovery and Data Mining, pp. 1125-1134, 2009.

[25] Soo-Min Kim and Eduard Hovy, "Determining the Sentiment of Opinions", Proceedings of $20^{\text {th }}$ International Conference on Computational Linguistics, pp. 1-7, 2004.

[26] Alistair Kennedy and Diana Inkpen, "Sentiment Classification of Movie Reviews using Contextual Valence Shifters", Computational Intelligence, Vol. 22, No. 2, pp. 110-125, 2006.

[27] Peter D. Turney and Michael L. Littman, "Measuring Praise and Criticism: Inference of Semantic Orientation from Association", ACM Transactions on Information Systems, Vol. 21, No. 4, pp. 315-346, 2003.

[28] Theresa Wilson, Janyce Wiebe and Paul Hoffmann, "Recognizing Contextual Polarity: An Exploration of Features for Phrase-Level Sentiment Analysis", Computational Linguistics, Vol. 35, No. 3, pp. 399-433, 2009.

[29] Kushal Dave, Steve Lawrence and David M. Pennock, "Mining the Peanut Gallery: Opinion Extraction and Semantic Classification of Product Reviews", Proceedings of $12^{\text {th }}$ International Conference on World Wide Web, pp. 519-528, 2003.

[30] J. Yi, T. Nasukawa, R. Bunescu and W. Niblack, "Sentiment Analyzer: Extracting Sentiments about a Given Topic using Natural Language Processing Techniques", Proceedings of $3^{\text {rd }}$ IEEE International Conference on Data Mining, pp. 1-8, 2003.

[31] Satoshi Morinaga et al., "Mining Product Reputations on the Web", Proceedings of $8^{\text {th }}$ ACM International Conference on Knowledge Discovery and Data Mining, pp. 341-349, 2002.

[32] Simon Tong and Daphne Koller, "Support Vector Machine Active Learning with Applications to Text Classification", Journal of Machine Learning Research, Vol. 2, pp. 45-66, 2001.

[33] Janyce M. Wiebe, "Learning Subjective Adjectives from Corpora", Proceedings of International Conference on American Association for Artificial Intelligence, pp. 1-6, 2000.

[34] Erik Cambria and Bebo White, "Jumping NLP Curves: A Review of Natural Language Processing Research", IEEE Computational Intelligence Magazine, Vol. 9, No. 2, pp. 4857, 2014.

[35] J. Yi, T. Nasukawa, R. Bunescu and W. Niblack, "Sentiment Analyzer: Extracting Sentiments about A given Topic using Natural Language Processing Techniques", Proceedings of $3^{r d}$ IEEE International Conference on Data Mining, pp. 1-8, 2003.

[36] Rui Xia et al., "Dual Training and Dual Prediction for Polarity Classification", Proceedings of $51^{\text {st }}$ Annual Meeting of the Association for Computational Linguistics, pp. 521525, 2013.

[37] Erik Cambria, "Affective Computing and Sentiment Analysis”, IEEE Intelligent Systems, Vol. 31, No. 2, pp. 102107, 2016.

[38] Bo Pang and Lillian Lee, "Opinion Mining and Sentiment Analysis", Foundations and Trends in Information Retrieval, Vol. 2, No. 1-2, pp. 1-135, 2008.

[39] Erik Cambria, Daniel Olsher and Dheeraj Rajagopal, "SenticNet 3: A Common and Common-Sense Knowledge base for Cognition-Driven Sentiment Analysis", Proceedings of $28^{\text {th }}$ Conference on Artificial Intelligence, pp. 1515-1521, 2014.

[40] Bo Pang and Lillian Lee, "Seeing Stars: Exploiting Class Relationships for Sentiment Categorization with Respect to Rating Scales", Proceedings of $43^{\text {rd }}$ Annual Meeting on Association for Computational Linguistics, pp. 115-124, 2005.

[41] Hassan Saif, Yulan He and Harith Alani, "Alleviating Data Sparsity for Twitter Sentiment Analysis", Proceedings of CEUR Workshop, pp. 1-8, 2012.

[42] Mike Thelwall, Kevan Buckley, Georgios Paltoglou, Di Cai and Arvid Kappas, "Sentiment Strength Detection in Short Informal Text", Journal of the American Society for Information Science and Technology, Vol. 61, No. 12, pp. 2544-2558, 2010. 